\title{
Labyrinthe
}

\section{La Kulturkritik et la constitution de la sociologie allemande : Ferdinand Tönnies, Georg Simmel et Max Weber}

\section{Aurélien Berlan}

\section{(2) OpenEdition \\ Journals}

Édition électronique

URL : http://journals.openedition.org/labyrinthe/1168

DOI : $10.4000 /$ labyrinthe.1168

ISSN : 1950-6031

Éditeur

Hermann

\section{Édition imprimée}

Date de publication : 15 janvier 2006

Pagination : 101-105

ISBN : 2-9526131-0-9

\section{Référence électronique}

Aurélien Berlan, « La Kulturkritik et la constitution de la sociologie allemande : Ferdinand Tönnies, Georg Simmel et Max Weber », Labyrinthe [En ligne], 23 | 2006 (1), mis en ligne le 24 juillet 2008, consulté le 22 avril 2019. URL : http://journals.openedition.org/labyrinthe/1168 ; DOI : 10.4000/ labyrinthe. 1168 


\section{LA KULTURKRITIK ET LA CONSTITUTION DE LA SOCIOLOGIE ALLEMANDE: Ferdinand Tönnies, Georg Simmel et Max Weber*}

Aurélien BERLAN aberlan@freesurf.fr

Par rapport aux sciences sociales qui se présentent sous la forme d'une réflexion empirique, la philosophie sociale et politique se donne une tâche normative: penser les normes et les idéaux qu'il serait possible et souhaitable d'incarner dans la réalité historique - normes et idéaux au nom desquels il est légitime de critiquer son état actuel. Depuis longtemps et de manière croissante, elle prend la forme de «théories de la justice» et présuppose donc que la critique du monde dans lequel on vit revient toujours à en dénoncer les injustices. Mais il ne semble pas que ce modèle permette de penser tous les discours critiques portant sur le présent.

L'aliénation et l'anomie, la déshumanisation des relations humaines et l'esseulement de l'homme dans la société moderne, l'uniformisation du monde, sa «marchandisation» ou sa dégradation (par exemple écologique) ne sont pas des « injustices». Il ne s'agit pas de problèmes quantitatifs touchant à l'inégale répartition des « biens» (matériels ou non: droits, honneurs, etc.) entre les hommes, mais de «maux» ou de «pathologies» susceptibles d'être violemment ressentis par chacun de nous. On se trouve ici face à une critique qui s'attaque à des conditions de vie conçues comme malsaines, nuisibles et dégradantes, et qui se réfère alors implicitement à une conception qualitative de la vie humaine, c'est-àdire à des théories de la vie «bonne ou saine».

Historiquement, ce modèle de critique est relié de manière étroite et ambivalente à la modernité. Il s'agit d'un discours typiquement moderne

\footnotetext{
* Thèse de philosophie sociale et d'épistémologie des sciences humaines en cours de réalisation dans le cadre d'une cotutelle franco-allemande, sous la direction de Catherine Colliot-Thélène (université de Rennes I) et de Axel Honneth (J. W. Goethe-Universität, Francfort-sur-le-Main).
} 
puisqu'il s'attaque aux effets négatifs de la modernisation impulsée par le développement économique capitaliste. En même temps, il s'agit d'une critique de la modernité qui non seulement peut conduire politiquement à des considérations antimodernes, mais qui surtout semble présupposer philosophiquement des formes prémodernes de justification. En effet, la modernité normative définie par Kant se caractérise précisément par la priorité libérale de la question de la «justice», dont on estime qu'elle est susceptible de trouver une solution rationnelle et universalisable, sur la question antique de la «vie bonne et heureuse» qui est, elle, renvoyée à l'arbitraire des préférences subjectives et à la contingence des formes de vie historiques. Nous sommes «au rouet»: la critique des pathologies de la modernité est constitutive de la réflexion moderne tout en débordant ses cadres normatifs. De droit, elle est indispensable $e t$ injustifiable; de fait, elle est inévitable et inavouable.

La critique des pathologies croise la réflexion sociale et la philosophie morale. L'objet de ma thèse est de développer cette problématique dans le cadre d'une archéologie de la sociologie allemande. Cette dernière s'est constituée à une époque où le discours qui critique la modernité - dont on retrouve certes des éléments chez Rousseau et les romantiques - tend à se généraliser sous la forme de la Kulturkritik, une vaste mouvance qui regroupe des philosophes (Nietzsche ${ }^{1}$, Dilthey, Ludwig Klages, Theodor Lessing, Rudolf Euken), des écrivains (Thomas Mann, Robert Musil), des poètes (Hugo von Hofmannstahl, Georg Trakl, Stefan George et son cercle), des historiens (Jacob Burckhardt, Ernst von Lasaulx), des publicistes nationalistes (Julius Langbehn et Paul de Lagarde), etc. Tous partagent une même sensibilité critique vis-à-vis du monde industriel, s'insurgent contre la religion du progrès et le rationalisme sous sa forme scientiste et utilitariste, et s'inquiètent quant au destin de l'humanité dans un monde dominé par un développement économique devenu autonome.

C'est dans ce contexte que la sociologie allemande a été fondée par Ferdinand Tönnies (1855-1939), Georg Simmel (1858-1918) et Max Weber (1864-1920). Elle y a pris la forme d'une «critique culturelle». Ces auteurs ont en effet en commun de faire de la sociologie une science critique du présent qui se caractérise par un double programme.

1. On estime en général que Nietzsche est la figure emblématique de la Kulturkritik. 


\section{La «Kulturkritik» et la constitution de la sociologie allemande}

D'une part, il s'agit de faire un diagnostic de leur époque, et c'est alors, grosso modo, le même cortège de pathologies qui est dénoncé: perte de sens et perte de liberté, aliénation et réification, déshumanisation et dépersonnalisation. Les nouvelles conditions de vie se répercutent sur l'économie psychique des individus: l'intellect calculateur prend le pas sur les autres dispositions de l'homme, notamment sur les facultés morales de sentir et de juger - facultés rendues superflues par une organisation sociale n' offrant plus de prise aux individus réduits à n'être que les rouages d'une machine qui les dépasse et les contraint. L'avenir appartient aux «experts sans esprit» et aux « hédonistes sans cœur» ${ }^{2}$.

D'autre part, il s'agit de comprendre le processus qui a mené à une telle situation: la modernisation est pensée à travers le paradigme de la rationalisation, qui n'est pas une réalisation heureuse de la raison dans l'histoire, mais, au contraire, une réification tragique, dans l'organisation sociale, de la rationalité formelle et instrumentale de l'entendement. C'est le passage de la communauté à la société (Tönnies), l'universalisation de la logique monétaire (Simmel), la bureaucratisation et le désenchantement du monde (Weber).

Les «pères fondateurs» de la sociologie allemande héritent foncièrement des thèmes et du ton pessimiste de la Kulturkritik. Les pages où Weber évoque la «cage d'acier ${ }^{3}$ » de la civilisation moderne sont, de ce point de vue, très caractéristiques. Mais Weber prend immédiatement ses distances par rapport à la critique culturelle qu'il vient d'énoncer, afin d'affirmer l'objectivité de son étude socio-historique ${ }^{4}$. D' autres prises de position de Weber, Simmel et Tönnies, témoignent de cette volonté de se démarquer, sur le plan méthodologique et politique, d'un discours plus «littéraire» que «scientifique» qui tendait à idéaliser le passé. Aussi serait-il réducteur d'assimiler complètement ces sociologues à la Kulturkritik. Il s'agit, au contraire, d'analyser les déplacements par lesquels ils vont reformuler sur le terrain théorique les inquiétudes de leur

2. Max Weber, L'Éthique protestante et l'esprit du capitalisme [1905], Paris, Plon, 1994, p. 225.

3. Ibidem, p. 220 sqq. La «cage d'acier» désigne la perte de liberté vécue par l'individu dans le « cosmos réifié» (ibidem) de la modernité. Ce qui était à l'origine le choix éthique des protestants (vouer sa vie au travail) est devenu une nécessité impérieuse. Plus généralement, la modernisation conduit à une différenciation des sphères d'activité (économie, politique, etc.) qui imposent leur logique propre à des acteurs qui n'ont guère d'autre choix que d'y obéir. Aussi l'entrepreneur, quelles que soient ses convictions sociopolitiques, est-il obligé de suivre la concurrence, quelles qu'en soient les conséquences sociales et humaines...

4. Ibidem, p. 225 : «Mais nous voici dans le domaine des jugements de valeur et de foi...» 
époque. Comment passer des intuitions d'une sensibilité commune à un diagnostic fondé en raison?

Dans ce processus dynamique de constitution, la notion de culture joue un rôle considérable sur trois plans. Elle définit tout d'abord une méthodologie antinaturaliste. Si les relations de la sociologie avec la Kulturkritik sont loin d'être univoques, c'est notamment parce que cette dernière avait une forte orientation antiscientifique et antisociologique. Les sociologues ont fondé une sociologie qui est une critique culturelle, mais ils l'ont fondée comme science dans un climat d'hostilité à la science en général et à la sociologie en particulier, cette dernière étant associée au positivisme de Comte et à l'utilitarisme de Mill - autant d'expressions typiques de ce rationalisme que la Kulturkritik maudissait. Les pionniers de la sociologie allemande ont donc dû construire une sociologie nouvelle qui puisse intégrer, contre l'optimisme rationaliste, les thèmes de la Kulturkritik, mais dont la méthode ne puisse prêter le flanc à la critique du naturalisme. C'est dans l'épistémologie des «sciences de la culture » qu'ils ont trouvé les instruments conceptuels pour transformer la «physique sociale» en ce qui se présente plutôt comme une «herméneutique culturelle» : une réflexion sur la signification pour l'humanité des mutations historiques.

On en arrive alors au second point: la notion de culture définit un domaine d'investigation plus large que le «social» conçu comme entité sui generis, un domaine dont la sociologie hérite par la tradition de la Kulturgeschichte (histoire des civilisations) et par la philosophie de Nietzsche qui écrivait: «Au lieu de la "société", le complexe de Kultur, mon principal objet d'intérêt ${ }^{5} »$. Enfin, si la critique est dite culturelle, c'est parce que le monde moderne est évalué à l'aune des dispositions qu'il « cultive» chez les hommes. La référence à la culture désigne donc une méthode originale d'investigation: comment les contextes culturels orientent-ils la genèse des caractères, influencent-ils le développement des dispositions, en inhibent-ils certaines et en favorisent-ils d'autres?

En posant cette question, la sociologie allemande reprend sur un mode empirique une problématique qui parcourt la philosophie de Platon à Montesquieu, celle du rapport entre les institutions et les mœurs que

5. Nietzsche Werke. Kritische Gesamtausgabe, Colli-Montinari (éd.), Walter de Gruyter, Berlin 1970, vol. VIII 2 , fragment 10 [28], p. 136. 
les premières présupposent et déterminent ${ }^{6}$. L'originalité des sociologues consiste à étendre cette problématique, originellement focalisée sur les institutions politiques, à l'ensemble des conditions de vie. Cet élargissement sanctionne une expérience nouvelle qui est à la racine de la Kulturkritik et de la sociologie allemande: la Révolution industrielle qui, à l'image de la révolution néolithique, constitue un immense bouleversement des conditions de vie humaine - un bouleversement qui a été d'autant plus brutal en Allemagne que l'industrialisation s'y est faite tardivement et rapidement.

On comprend dès lors la spécificité de la sociologie allemande, notamment par rapport à la sociologie française. Si cette dernière a été fondée au début du XIX ${ }^{\mathrm{e}}$ siècle pour penser une révolution d'origine politique (1789), la sociologie allemande s'est constituée en réponse à une révolution d'origine économique. Alors que celle-là redoute l'anarchie révolutionnaire tout en voulant sauver le progrès (que l'on songe à la devise de Comte, inventeur du terme de sociologie « ordre et progrès »), la seconde met en question ce «progrès» impulsé par l'industrie, ainsi que l'ordre étouffant qu'il met en place, au nom d'un idéal d'existence que les nouvelles conditions de vie semblent menacer. Elle contribue ainsi à un retour critique de la modernité sur elle-même - une problématique qui semble toujours plus actuelle.

6. Que l'on pense au parallélisme entre les constitutions et les types humains chez Platon (République $I X)$ ou au lien entre telle constitution et telle passion dominante chez Montesquieu (L'Esprit des lois). 